\title{
Specific and cross-reacting monoclonal antibodies to Bordetella parapertussis and Bordetella bronchiseptica lipopolysaccharides
}

\author{
Karine Le Blay, ${ }^{1}$ Martine Caroff, ${ }^{1}$ James C. Richards, ${ }^{2}$ Malcolm B. Perry ${ }^{2}$ \\ and Richard Chaby'
}

Author for correspondence: Richard Chaby. Tel: +331694171 91. Fax: +33169853715.

1 Equipe 'Endotoxines', URA-1116 du CNRS, Université de Paris-Sud, 91405 Orsay, France

2 Institute for Biological Sciences, National Research Council of Canada, Ottawa, Ontario Canada

\begin{abstract}
Three groups of monoclonal antibodies (mAbs) were produced that would be useful for immunochemical typing and diagnosis of infections due to Bordetella species, and for the structural analysis of their lipopolysaccharides. PP6, a representative of the first group, recognizes an epitope shared by smooth-type Bordetella parapertussis and Bordetella bronchiseptica lipopolysaccharides (LPS). This epitope is carried by structurally identical polymeric 0-chains (POC) present on both LPS molecules. PP8 and PP9 are representatives of the second group of mAbs. The interaction of PP8 and PP9 with B. parapertussis and B. bronchiseptica LPS requires POC, but periodatesensitive sugar units of the core are also involved in the binding. The mAb BRg1 belongs to the third group, and specifically recognizes B. bronchiseptica LPS. Binding and inhibition studies with various Bordetella LPS molecules, and with their polysaccharide fragments, indicated that BRg1 interacts with a structure located at the hinge between the POC and a core region of the $B$. bronchiseptica LPS containing periodate-resistant sugars. This suggests that the structures of the hinge regions of the $B$. parapertussis and $B$. bronchiseptica LPS are different.
\end{abstract}

Keywords: Bordetella bronchiseptica, Bordetella parapertussis, lipopolysaccharides, monoclonal antibodies

\section{INTRODUCTION}

Whooping cough is a particularly severe disease of infants, characterized by repeated bouts of paroxysmal coughing. The disease is caused by Bordetella pertussis which, in the early phase, attaches to and multiplies on the cilia of the epithelial cells of the respiratory tract (Pittman, 1984; Novotny, 1990). Other members of the genus Bordetella, namely B. parapertussis, B. bronchiseptica, and B. avium, are taxonomically strongly related to $B$. pertussis (Goodnow, 1980; Musser et al., 1986) and can induce respiratory diseases in different hosts. B. parapertussis is a human pathogen that causes a whooping cough syndrome that is milder than that normally seen with B. pertussis (Novotny, 1990 ), probably because it does not produce the pertussis toxin (Monack et al., 1989). B. bronchiseptica is a respiratory tract pathogen of mammals, which affects a number of

\footnotetext{
Abbreviations: i.p., intraperitoneal; i.v., intravenous; $m A b$, monoclonal antibody; nad-PS, polysaccharide released by nitrous acid deamination; POC, polymeric O-chain fragment; PS, polysaccharide released by acid hydrolysis.
}

farm animals (Goodnow, 1980), and has been reported to cause bacteraemia in immunocompromized humans (Bauwens et al., 1992; Katzenstein et al., 1984). B. avium is the aetiological agent of rhinotracheitis of birds (Kersters et al., 1984). The disease in birds has a number of similarities to pertussis in humans (Simmons et al., 1979), including the fact that young birds are very susceptible, while older birds are refractory to the infection (Simmons \& Gray, 1979).

The pathogenesis of Bordetella infections is not fully understood, but it is believed that several substances released by the bacteria (toxins, haemagglutinin, adenylate cyclase) contribute to the manifestations of the disease (Weiss \& Hewlett, 1986). Among these, lipopolysaccharide (LPS), a major exposed component of the outer membrane, has been implicated as a determinant of both virulence and immunity (Rietschel et al., 1982). Knowledge of the immunogenicity of the LPS of $B$. pertussis, B. parapertussis, and B. bronchiseptica is limited. Amano et al. (1990) showed that LPS from B. pertussis reacted with antisera prepared against $B$. pertussis and $B$. 
bronchiseptica, that LPS from $B$. parapertussis reacted with antisera to $B$. parapertussis and $B$. bronchiseptica, and that L.PS from $B$. bronchiseptica reacted with antisera raised against any of the three species. It was concluded that the L.PS molecules from $B$. pertussis and B. parapertussis have no common structure, whereas each has a common structure with the LPS from B. bronchiseptica. A recent study (Di Fabio et al., 1992) demonstrated that smooth I.PS from B. parapertussis and B. bronchiseptica have identical O-polysaccharide chains consisting of linear unbranched polymers of $(1 \rightarrow 4)$-linked 2,3-diacetamido2,3 -dideoxy- $\alpha$-L-galactopyranosyluronic acid residues, whereas their core oligosaccharide components are different. Lugtenberg et al. (1984) showed that LPS from B. bronchiseptica contains both smooth (LPS-I) and rough (I.PS-II) fractions, detectable by SDS-PAGE, and suggested that LPS-I is a protective antigen. The aim of the present study was to produce and characterize monoclonal antibodies directed against the common antigenic $\mathrm{O}$-chain of $B$. parapertussis and B. bronchiseptica, in view of their potential use in passive protection, or as diagnostic reagents.

\section{METHODS}

Bacterial strains and growth conditions. Cells of $B$. broncbiseptica (strain NRCC 4170 from the National Research Council Veterinary Collection) were grown in 70 litre fermenters using $3.7 \%(\mathrm{v} / \mathrm{v})$ brain heart infusion containing $5 \%(\mathrm{v} / \mathrm{v})$ horse serum, at $37^{\circ} \mathrm{C}, 2000$ r.p.m. with aeration, for 18 h. Cells of $B$. parapertussis (strain ATCC 15989 from the American Type Culture Collection; and rough mutant strain NRCC 4424, original designation 504-2R, kindly supplied by Dr M. Peppler, University of Alberta, Edmonton, Canada) were grown for $72 \mathrm{~h}$ with rotary agitation in the above media at $37^{\circ} \mathrm{C}$, using 4 litre baffled conical flasks. All cells were killed with $2 \%(\mathrm{v} / \mathrm{v})$ phenol (final concentration) and were collected by centrifugation.

Chemicals and reagents. Tween 20, 2,6,10,16-tetramethylpentadecane (pristane), horseradish peroxidase-labelled goat anti-mouse $\mathrm{Ig}$, anti-mouse $\mathrm{H}$ chain of different classes and subclasses, and the peroxidase substrate o-phenylenediamine dihydrochloride, were from Sigma. Gelatin was from Rousselot Kuhlmann. Polyethylene glycol 1500 was from Boehringer Mannheim.

Preparation of LPS and derived fragments. The LPS from Bordetella pertussis was isolated by the phenol/water extraction procedure, and purified as described previously (Le Dur et al., 1980). Lipid A was isolated from the B. pertussis LPS by mild acid hydrolysis (Caroff et al., 1988). LPS from B. bronchiseptica and B. parapertussis was prepared by extraction of the cells by the modified enzyme/phenol/water method and ultracentrifugation of the concentrated, dialysed, aqueous phases $(105000 \mathrm{~g}$, $4{ }^{\circ} \mathrm{C}, 12 \mathrm{~h}$ ) (Johnson \& Perry, 1976). The intact polysaccharide fragment (PS) of the B. bronchiseptica LPS was isolated by Sephadex G-50 chromatography, after hydrolysis of a $1.5 \%$ $(\mathrm{w} / \mathrm{v})$ solution of the LPS in sodium acetate $(\mathrm{pH} 4.5)$ for $2 \mathrm{~h}$ at $100^{\circ} \mathrm{C}$ in the presence of $1 \%(\mathrm{w} / \mathrm{v}) \mathrm{SDS}$ (Caroff et al., 1988). The polymeric $\mathrm{O}$-chain fragment (POC) of the B. bronchiseptica LPS was isolated by Sephadex G-50 chromatography after a Smith-type degradation of the LPS (Goldstein et al., 1970), consisting of a sequence of treatments involving oxidation with sodium periodate $\left(1 \%(\mathrm{w} / \mathrm{v}), 20^{\circ} \mathrm{C}, 2 \mathrm{~d}\right)$, reduction with sodium borohydride $(0.6 \%, 6 \mathrm{~h})$, and acid hydrolysis $(2 \%, \mathrm{v} / \mathrm{v}$, acetic acid, $100^{\circ} \mathrm{C}, 2 \mathrm{~h}$ ). Several sugars of the core region, containing vicinal diols, are susceptible to this treatment, whereas the polymeric O-chain, consisting of $(1 \rightarrow 4)$-linked 2,3-diacetamido-2,3-dideoxy- $\boldsymbol{\alpha}$ - $\mathrm{L}$-galactopyranosyluronic acid residues, is resistant. According to the Bradford assay (Bradford, 1976) the protein content of the POC preparation was less than $0.03 \%$. The nad-PS fragment of the B. bronchiseptica LPS was isolated by Sephadex G50 chromatography after nitrous acid cleavage, consisting of incubation of the LPS for $4 \mathrm{~h}$ at $20^{\circ} \mathrm{C}$ in a freshly prepared mixture of water $/ 5 \%(\mathrm{w} / \mathrm{v})$ sodium nitrite $/ 30 \%$ (v/v) acetic acid (1:1:1, by vol.), as described previously (Caroff et al., 1990; Deprun et al., 1993). Analyses of the nad-PS preparation by thin-layer chromatography (Caroff \& Karibian, 1990) showed no detectable contaminants.

Preparation of the POC-BSA conjugate. The PS fragment of the B. bronchiseptica LPS $(150 \mathrm{mg})$ was treated with $0.1 \mathrm{M}$ sodium metaperiodate $(4 \mathrm{ml})$ for $4 \mathrm{~d}$ at $20^{\circ} \mathrm{C}$. The excess periodate was removed by extensive dialysis against distilled water and the retained solution $(10 \mathrm{ml})$ was made up to $1.5 \%$ $(\mathrm{v} / \mathrm{v})$ acetic acid and was kept at $100^{\circ} \mathrm{C}$ for $30 \mathrm{~min}$. The cooled solution was subjected to Sephadex G-50 gel filtration and the collected polysaccharide was lyophilized. The isolated product (activated POC) consists of intact polymeric $\mathrm{O}$-chains linked to a degraded core containing aldehydo groups suitable for conjugation to carrier protein. The activated POC $(80 \mathrm{mg})$ and BSA (40 mg) were dissolved in $0.1 \mathrm{M}$ aqueous sodium bicarbonate $(4 \mathrm{ml})$ and, following the addition of sodium cyanoborohydride $(100 \mathrm{mg})$, the stirred mixture was kept at $20^{\circ} \mathrm{C}$ for $7 \mathrm{~d}$. The resulting conjugate was purified by gelpermeation chromatography on a Bio-gel A $0.5 \mathrm{M}$ column $(100 \times 1 \mathrm{~cm})$ using $0.01 \mathrm{M}$ phosphate buffer $(\mathrm{pH} 7 \cdot 2)$ containing $9 \%(\mathrm{w} / \mathrm{v})$ sodium chloride. Chemical analyses and massspectrometry indicated that the POC-BSA conjugate contained about $24 \%$ (w/w) POC.

Animal immunizations. Groups of four BALB/c mice ( $R$. Janvier, Le Genest Saint-Isle, France) were immunized at days 1,14 and 28 with $1 \mu \mathrm{g}$ of LPS from $B$. parapertussis or $B$. bronchiseptica, or with $100 \mu \mathrm{g}$ (lyophilized dry wt) of phenolkilled organisms of $B$. parapertussis. Each preparation was suspended in $200 \mu \mathrm{l}$ nonpyrogenic $0.15 \mathrm{M} \mathrm{NaCl}$ solution (Biosedra, Malakoff, France), and injected i.v. Sera were collected $7 \mathrm{~d}$ after each injection.

Production of mAbs. The mouse which produced the highest titre of anti-LPS antibodies (detected after the second immunization) was selected for fusion. Three days after the last injection, the spleen cells were fused with SP2/0-Ag.14 myeloma cells (10:1 ratio), in $45 \%$ (w/v) PEG 1500 as described previously (Girard \& Chaby, 1985). Fusion products were cultured in a $50 \mu \mathrm{M}$ hypoxanthine $/ 10 \mu \mathrm{M}$ azaserine selection medium. Resulting colonies were tested for secretion of anti-LPS antibodies, and cloned by a limiting dilution technique (Nowinsky et al., 1979). Clones were expanded as ascites by injection $\left(5 \times 10^{6}\right.$ cells per mouse, i.p.) in BALB/c mice which had been treated with pristane $(0.5 \mathrm{ml}$, i.p.) two weeks previously.

ELISA and ELISA inhibition. Sera and cell culture supernatants were assayed for anti-LPS or anti-POC-BSA antibody specificity by ELISA. The 96-well Immulon-A plates (Greiner Labortechnik) were coated with the antigen $(100 \mu \mathrm{l}$ of $20 \mu \mathrm{g} \mathrm{ml}^{-1}$ suspensions in $50 \mathrm{mM}$ Tris $/ \mathrm{HCl}$ buffer plus $20 \mathrm{mM}$ $\mathrm{MgCl}_{2}, \mathrm{pH} 9.6$ ). After overnight incubation at $20^{\circ} \mathrm{C}$ under mild stirring, the unbound antigen was washed away with $0.1 \%$ Tween 20 in PBS (Tween buffer), and the plates were incubated for $1 \mathrm{~h}$ at $37^{\circ} \mathrm{C}$ with $0.25 \%$ gelatin in the same buffer. The plates were rewashed with this solution and a sample of serum or culture supernatant (diluted in the same solution) was added to each well. After incubation for $2 \mathrm{~h}$ at $37^{\circ} \mathrm{C}$, the plates were 
Table 1. mAbs produced after different immunizations

\begin{tabular}{|c|c|c|c|c|}
\hline \multicolumn{3}{|c|}{ Immunization } & \multicolumn{2}{|c|}{ Monoclonal antibody } \\
\hline $\begin{array}{l}\text { Material } \\
\text { injected* }\end{array}$ & Origin & $\begin{array}{c}\text { No. of } \\
\text { injections } \dagger\end{array}$ & Name & Class \\
\hline LPS & B. parapertussis & 3 & PP6 & $\operatorname{IgG} 3$ \\
\hline Cells & B. parapertussis & 2 & PP8 & $\operatorname{Ig} G 3$ \\
\hline Cells & B. parapertussis & 2 & PP9 & $\lg G 3$ \\
\hline LPS & B. bronchiseptica & 3 & BRg1 & $\operatorname{IgM}$ \\
\hline
\end{tabular}

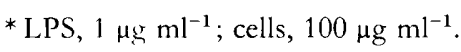

ti.v., at $14 \mathrm{~d}$ intervals.

washed with the Tween buffer, and the peroxidase-labelled goat anti-mouse Ig reagent added. After incubation at $37^{\circ} \mathrm{C}$ for $90 \mathrm{~min}$, the plates were washed and developed by adding $100 \mu \mathrm{l}$ of a treshly prepared solution of 0 -phenylenediamine $\left(0.5 \mathrm{mg} \mathrm{ml}^{-1}\right)$ in a $0.05 \mathrm{M}$ phosphate/citrate buffer ( $\left.\mathrm{pH} 5\right)$ containing sodium perborate $(0.03 \%)$ as a substitute for hydrogen peroxide. After $15 \mathrm{~min}$ at room temperature, the reaction was stopped with a solution of $0.5 \%$ sodium sulphite in $2 \mathrm{M} \mathrm{H}_{2} \mathrm{SO}_{4}(50 \mu \mathrm{l}$ per well). The plates were scanned at $490 \mathrm{~nm}$ in a Dynatech MR 5000 spectrophotometer. Antibody isotypes were determined by the same ELISA procedure, by using peroxidase-labelled goat antibodies against mouse Ig of different classes and subclasses. In ELISA inhibition experiments, ascitic fluids $1 / 6000$ dilutions, unless otherwise specified) were preincubated for $1 \mathrm{~h}$ at $37^{\circ} \mathrm{C}$ with various concentrations of the inhibitors, in gelatin-coated wells. The solutions were then transfer red to wells coated with LPS (by overnight incubation at room temperature with $2 \mu \mathrm{g} \mathrm{ml}^{-1}$ LPS in the Tris/ $\mathrm{HCl}$ coating buffer). The binding of the antibodies to LPS was determined as described in the direct ELISA procedure.

\section{RESULTS}

\section{Production of hybridoma and ascitic fluids}

Mice were injected i.v., at $14 \mathrm{~d}$ intervals, either with LPS from $B$. parapertussis or B. bronchiseptica, or with phenolkilled B. parapertussis cells. In a preliminary experiment, animals were bled $7 \mathrm{~d}$ after each injection, and the reactivity of the sera against the homologous LPS was determined by ELISA. We found that the optimal antiLPS response of animals immunized with killed microorganisms was reached faster (second immunization) than that of animals immunized with LPS (third immunization) (results not shown). Fusion experiments were performed $3 \mathrm{~d}$ after the second injection with animals immunized with killed cells, and $3 \mathrm{~d}$ after the third injection with animals immunized with LPS. Hybridomas were screened first for their reactivity with the homologous LPS, and were rescreened for the absence of cross-reactivity with a Lipid A preparation obtained from the B. pertussis LPS. Among the remaining hybridomas, four clones of high reactivity with LPS were selected for this study ('Table 1). One clone (PP6) was produced with a mouse immunized with the B. parapertussis LPS, two clones (PP8 and PP9) were generated with animals immunized with killed $B$. parapertussis organisms, and one clone (BRg1) was

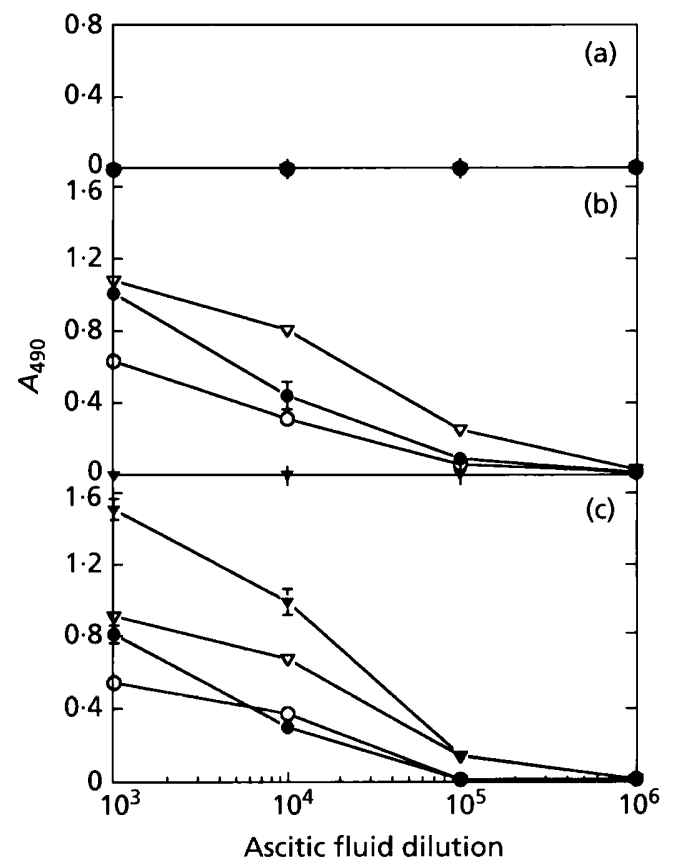

Fig. 1. Reactivity of mAbs with LPS from wild Bordetella strains. Plates were coated with LPS from $B$. pertussis (a), $B$. parapertussis (b), and B. bronchiseptica (c). The plates were incubated with different dilutions of ascitic fluids from mAbs PP6 (O), PP8 $(\circlearrowleft)$, PP9 $(\nabla)$ and BRg1 $(\nabla)$. The binding of the mAbs was determined by ELISA. Values represent the mean \pm SD of triplicate determinations.

obtained with a mouse immunized with the $B$. bronchiseptica LPS. PP6, PP8 and PP9 were IgG3, whereas BRg1 is an IgM (Table 1).

\section{Binding of the mAbs with different Bordetella LPSs}

The reactivity of PP6, PP8, PP9 and BRg1 with LPS from B. pertussis, B. parapertussis, and B. bronchiseptica was analysed by ELISA. The results (Fig. 1) show that none of these antibodies reacted with the B. pertussis LPS. BRg1 reacted exclusively with the $B$. bronchiseptica LPS, whereas PP6, PP8 and PP9 reacted with both $B$. parapertussis and $B$. bronchiseptica LPS. 


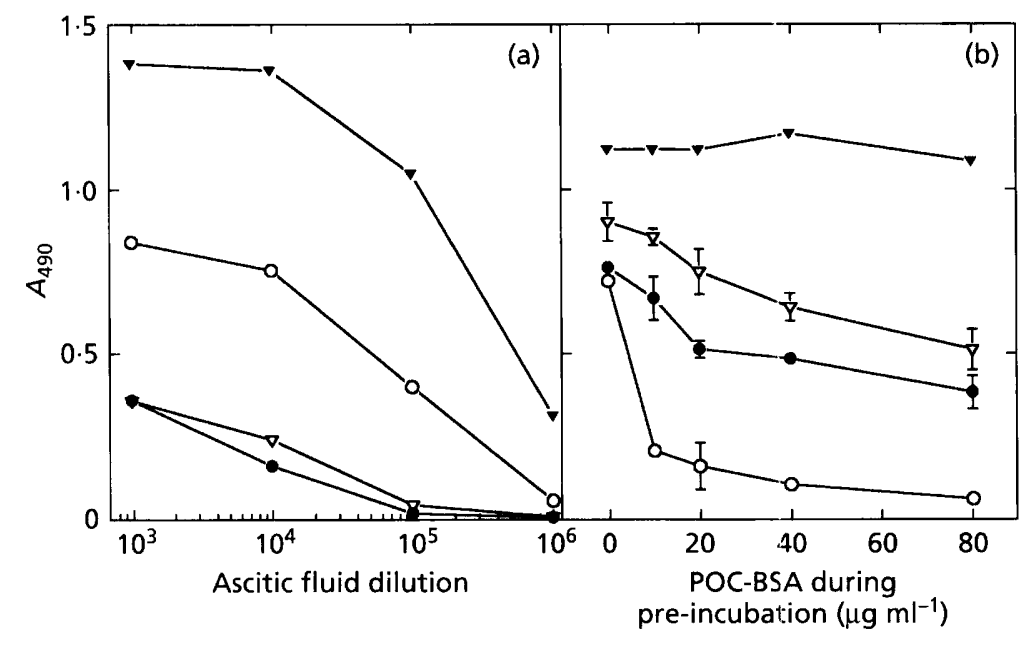

Fig. 2. Interaction of mAbs with POC-BSA. The antigen stuck to plastic (a) and in solution (b) was incubated with ascitic fluids from clones PP6 (O), PP8 (O), PP9 $(\nabla)$, and BRg1 $(\boldsymbol{\nabla})$. The interaction was determined by direct ELISA (a), and by ELISA inhibition with plates coated with the $B$. bronchiseptica LPS (b). Values represent the mean \pm SD of triplicate determinations.

\section{Reactivity of the mAbs with POC-BSA}

The existence of cross-reactivity of PP6, PP8 and PP9 with B. parapertussis and B. bronchiseptica LPS prompted us to examine their reactivity with the polymeric O-chains (POC) of the LPS, which have been reported to be structurally identical (Di Fabio et al., 1992). We used the conjugate POC-BSA prepared with POC isolated from the B. bronchiseptica LPS and coupled to BSA. The reactivities of the $\mathrm{mAbs}$ with POC-BSA were first examined by ELISA (Fig. 2a). As observed above with the B. bronchiseptica LPS, the four $\mathrm{mAbs}$ bound to POCBSA-coated plates. The binding of $\mathrm{mAbs} \mathrm{PP} 8$ and PP9 to POC-BSA was however significantly lower than that to the B. bronchiseptica LPS. None of the mAbs reacted with unsubstituted BSA (results not shown).

In order to analyse the interactions of the $\mathrm{mAbs}$ with POC-BSA in fluid phase, the mAbs $(1 / 6000$ dilution of ascitic fluids) were preincubated with various concentrations (up to $80 \mu \mathrm{g} \mathrm{ml}^{-1}$ ) of POC-BSA, and the resulting inhibition of binding of the $\mathrm{mAbs}$ to plates coated with $B$. bronchiseptica LPS was determined by ELISA. The results (Fig. 2b) show that the interaction of PP6 with POC-BSA in fluid phase efficiently and completely inhibited the binding to the B. broncbiseptica LPS. Only partial inhibition was obtained with the PP8 and PP9 antibodies, and inhibition was not observed with BRg1.

\section{Reactivity of the antibodies with isolated polysaccharides}

The discrepancy between the reactivity of BRg1 with POC-BSA coated on plastic (Fig. 2a), and the absence of reactivity with POC-BSA in fluid phase (Fig. 2b), prompted us to examine the interaction of BRg1 with the isolated polysaccharide fragment (not linked to BSA) under the latter conditions. A PS preparation isolated from the $B$. broncbiseptica LPS under mild hydrolytic conditions and the POC fragment isolated from PS after partial destruction of the core moiety by a Smith degradation, were preincubated with BRg1. The inhibition of the binding of BRg1 to plates coated with the $E$.

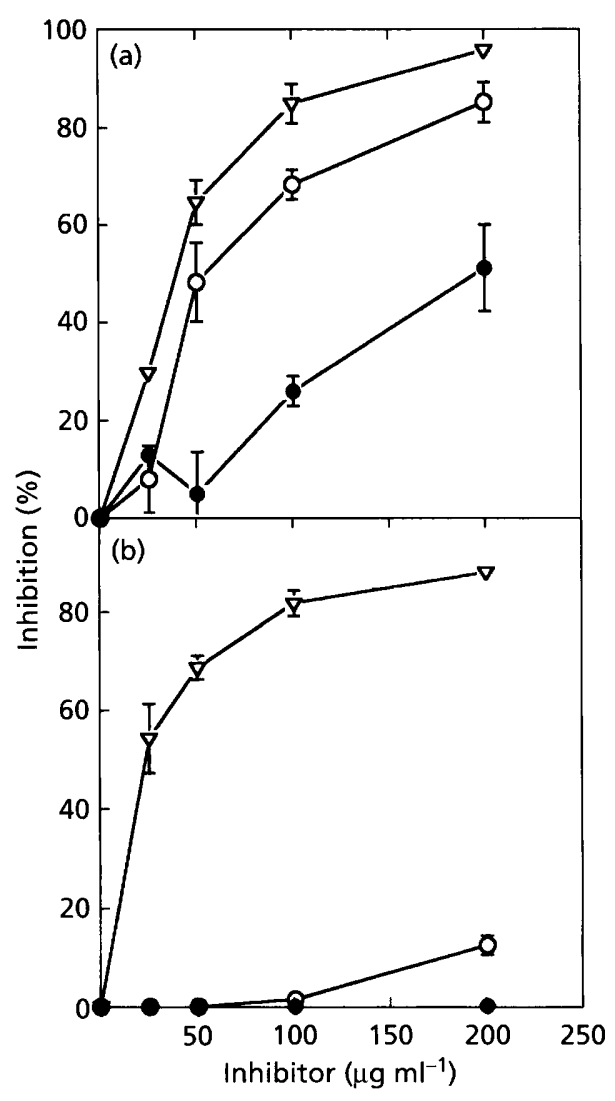

Fig. 3. Reactivity of PP8 and BRg1 with antigens in fluid phase. PP8 (ascites dilution 1/6000) (a) and BRg1 (ascites dilution $1 / 12000)(b)$ were incubated with solutions of LPS $(\nabla)$, PS $(\bigcirc)$, and POC (O), at various concentrations. Reactivities were determined by ELISA inhibition, with plates coated with the $B$. bronchiseptica LPS. Values represent the mean \pm SD of triplicate determinations.

bronchiseptica LPS was compared to that of PP8. The results (Fig. 3) show that the binding of BRg1 was not inhibited by POC, was slightly inhibited by high concentrations of PS $\left(200 \mu \mathrm{g} \mathrm{ml}^{-1}\right)$ and was very efficiently 


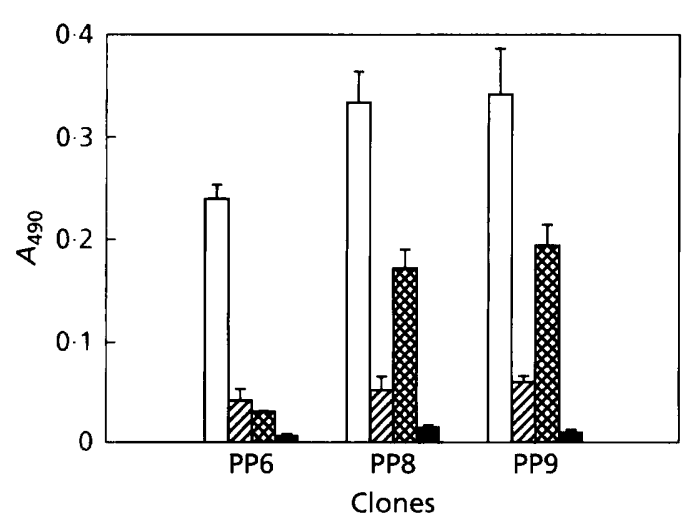

Fig. 4. Reactivity of PP6, PP8 and PP9 with antigens in fluid phase. Ascites (1/12000 dilution) were incubated with buffer alone $(\square)$, or with solutions $\left(200 \mu \mathrm{g} \mathrm{ml}^{-1}\right)$ of PS (Z), POC (国), and LPS ( $\boldsymbol{\square})$. Reactivities were determined by ELISA inhibition, with plates coated with $B$. bronchiseptica LPS. Values represent the mean \pm SD of triplicate determinations.

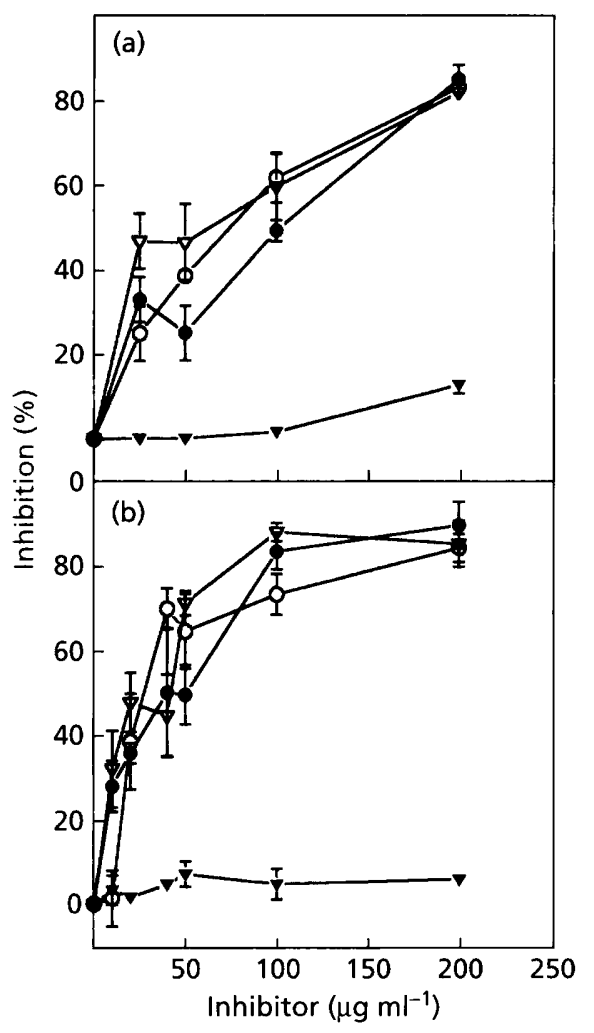

Fig. 5. Reactivity of the mAbs with PS and nad-PS. PP6 (O), PP8 $(0)$ PP9 $(\nabla)$ and BRg1 ( $\nabla)$ (ascites dilution 1/6000) were incubated with various concentrations of PS (a) and nad-PS (b). Reactivities were determined by ELISA inhibition, with plates coated with $B$. bronchiseptica LPS. Values represent the mean \pm SD of triplicate determinations.

inhibited by the intact LPS. On the other hand, the binding of PP8 was partially (50\%) inhibited with POC, and almost completely inhibited with PS and LPS.

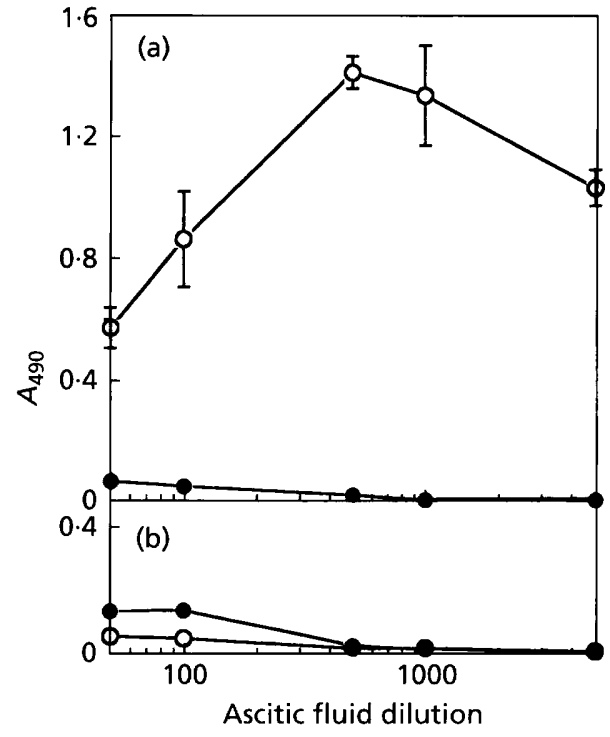

Fig. 6. Reactivity of PP8 and BRg1 with smooth- and rough-type B. parapertussis LPS. Plates coated with smooth (a) and rough (b) B. parapertussis LPS were incubated with different dilutions of ascitic fluids from clones PP8 (O) and BRg1 (O). The binding of the mAbs was determined by ELISA. Values represent the mean $\pm S D$ of triplicate determinations.

$\mathrm{mAb}$ PP9 behaved in the same way as PP8 in this test (Fig. 4). At $200 \mu \mathrm{g}$ POC ml ${ }^{-1}$, only partial (50\%) inhibition of binding of PP8 and PP9 was observed. On the other hand, the specificity of PP6 appeared different under these conditions, since POC almost completely inhibited the binding of this $\mathrm{mAb}$ to the B. bronchiseptica LPS.

Since certain sugar units belonging to the core region of the B. bronchiseptica LPS might be resistant to periodate oxidation, and thus might be present in POC and POC$\mathrm{BSA}$, we prepared another polysaccharide fraction containing the O-chain. This polysaccharide (nad-PS) was released from the B. bronchiseptica LPS by nitrous acid cleavage of the glycosidic linkage of 2-amino sugars with free $\mathrm{NH}_{2}$ groups present in the core. We analysed the interactions of the mAbs with nad-PS in fluid phase, and compared them to the interactions with PS. The results (Fig. 5) show that the interactions with PS and nad-PS are very similar, which indicates that the amino sugar units that are sensitive to nitrous acid treatment (and other sugar units that remain linked to lipid A after this treatment) are not involved in the epitopes recognized by PP6, PP8, and PP9. The absence of reactivity of BRg1 in fluid phase with the polysaccharide fragment released by acid hydrolysis (PS) (Fig. 3b and Fig. 5a) was also observed with the polysaccharide fragment released by nitrous acid deamination (nad-PS) (Fig. 5b).

\section{Reactivity of BRg1 with LPS from rough $B$. parapertussis}

Antigenic differences in the core regions of $B$. parapertussis and $B$. bronchiseptica LPS may explain the absence of reactivity of BRg1 with the former. A rough mutant of $B$. 
parapertussis is now available. The polysaccharide region of its LPS is restricted to the core. The reactivity of BR g1 with the smooth- and rough-type LPS from $B$. parapertussis were compared by ELISA. The mAb PP8 was used as a reference in the same experiment. The results (Fig. 6) shows that at low antibody concentrations (less than 1/500) BRg1 does not interact either with the LPS from wild B. parapertussis, or with the LPS from the rough mutant, whereas PP8 interacts exclusively with the smooth-type LPS. The mAbs PP6 and PP9 behaved like PP8 in this test (results not shown). At higher antibody concentrations (more than 1/500) binding of BRg1 to both smooth- and rough-type LPS from $B$. parapertussis was detectable. This low level of binding with the $B$. parapertussis LPS was lower with the smooth than with the rough form, thus indicating a weak but significant reactivity of BRg1 with a core epitope of $B$. parapertussis LPS.

\section{DISCUSSION}

Members of the genus Bordetella are Gram-negative respiratory pathogens of a variety of animal species. $B$. pertussis is the aetiological agent of human whooping cough. The present study focused on two other members of the genus Bordetella, B. broncbiseptica and B. parapertussis. Although it is generally thought that $B$. bronchiseptica primarily infects nonhuman mammals, this organism is sometimes a pathogen for humans (Bauwens et al., 1992; Katzenstein et al., 1984). B. parapertussis is a human pathogen which causes a whooping-cough-like respiratory disease that can hardly be distinguished from the $B$. pertussis infection by clinical criteria (Novotny, 1990). In view of the recent recrudescence of whooping cough symptoms in several developed countries, and of the limited number of epidemiological studies on human infections with $B$. parapertussis and B. bronchiseptica, the availability of well-characterized monoclonal antibodies allowing accurate typing and diagnosis would be of great interest in clinical and epidemiological studies on humans and animals.

Among various components exposed on the cell surface, the endotoxin (LPS) is a major antigenic constituent of these Gram-negative bacteria. mAbs specific for the ()chain and core moieties of LPS from other bacteria have proved useful for typing studies, and some of them have been reported to be protective against infection (Montaraz et al., 1986; Terashima et al., 1991). In the present study, after immunization of mice with killed $B$. parapertussis cells, or with LPS extracted from B. parapertussis and $B$. bronchiseptica, we prepared three groups of monoclonal antibodies. PP6, PP8 and PP9 are representatives of two groups of antibodies that cross-react with $B$. parapertussis and $B$. bronchiseptica LPS, but do not react with the $B$. pertussis LPS (Fig. 1). The epitopes that are recognized by these antibodies are clearly carried by the polymeric $\mathrm{O}$ chain of these smooth-type LPSs, since the antibodies interacted with POC-BSA (either coated on plastic, or as a soluble material), whereas they did not bind to the rough-type LPS from $B$. parapertussis. However, inhi- bition experiments with POC-BSA (Fig. 2b) and POC (Fig. 4) indicated that the specificities of PP8 and PP9, which are very similar (both are incompletely inhibited by POC-BSA and POC), are different from that of PP6 (which is completely inhibited by POC-BSA and POC). This observation suggests that one or more sugar units that are sensitive to the periodate treatment used to prepare POC and POC-BSA are involved in the epitope recognized by PP8 and $\mathrm{PP9}$, but are not involved in the epitope recognized by PP6.

The $\mathrm{mAb} B \mathrm{Bg} 1$ is a representative of a third group of antibodies that selectively reacted with the $B$. bronchiseptica LPS (Fig. 1). This selectivity indicates that BRg1 interacts with sugar units of the core region of the B. bronchiseptica LPS that are absent or differently substituted in $B$. parapertussis and B. pertussis LPS. Preliminary analyses indicated that no difference could be detected in the masses (plasma desorption mass spectrometry, PDMS) and chromatographic migrations (TLC) of dephosphorylated cores from B. pertussis and B. bronchiseptica LPS (M. Caroff and others, unpublished). This would suggest that the epitope which is recognized by BRg1 requires not only certain core constituents, but also the presence of the O-chain. This hypothesis is strengthened by the observation that BRg1 binds very efficiently to plastic coated with a POC-BSA conjugate (Fig. 2a). It should be noted that a prolonged treatment of the polysaccharide with sodium periodate was used during the preparation of POC-BSA. The reactivity of BRg1 with POC-BSA therefore suggests that BRg1 interacts with an epitope in which both the O-chain and core sugars resistant to periodate oxidation are involved. This epitope is probably located at the hinge between the O-chain and the core.

An apparent discrepancy in our results is that POC-BSA and POC were unable to inhibit the binding of BRg1 to the B. bronchiseptica LPS (Fig. 2b, Fig. 3b) whereas BRg1 was able to bind very efficiently to plates coated with POC-BSA (Fig. 2a). This observation that the reactivity of BRg1 with POC-BSA is very low when the antigen is in a soluble form, and high when the antigen is stuck on plastic, shows that the spatial conformation of the molecule has a critical influence on the presentation of the epitope recognized by BRg1. In fluid phase, the absence of reactivity of $\mathrm{BRg} 1$ with various polysaccharide fragments (PS, nad-PS, and POC) of the B. bronchiseptica LPS suggests that the lipid A region (or an acid-labile component) of the LPS plays a critical role in the conformation of the antigenic moiety of the molecule in solution.

It is noteworthy that clones secreting anti-O-chain antibodies were not produced during immunization of mice with the $B$. bronchiseptica LPS. This may indicate that the epitope present at the hinge region, which is recognized by $\mathrm{BRg} 1$, is more immunogenic than those of the O-chain. The failure to obtain similar clones upon immunization with killed cells is probably due to the fact that this imunogenic epitope is less accessible at the surface of the bacterium than on its isolated LPS. In this regard, the interaction of the mAbs with accessible cell 
surface epitopes, and the influence of this interaction on the viability of the bacteria, are important parameters addressing the issue of protection against infection. It has been reported (Archambault et al., 1991) that among $\mathrm{mAbs}$ which recognize $B$. pertussis LPS and cross-react with $B$. bronchiseptica, several are bactericidal for $B$. pertussis cells but none is bactericidal for $B$. bronchiseptica, even though they react with surface-accessible antigenic determinants. Whether the mAbs described in our study could protect against infection, or whether they could be used as diagnostic reagents will be evaluated further.

\section{ACKNOWLEDGEMENTS}

This work was supported by the Centre National de la Recherche Scientifique (Mission des Relations Internationales). We would like to thank Dr M. Peppler for providing the rough B. parapertussis strain and Mr D. W. Griffith for the production of the bacterial cells.

\section{REFERENCES}

Amano, K.-I., Fukushi, K. \& Watanabe, M. (1990). Biochemical and immunological comparison of lipopolysaccharides from Bordetella species. I Gen Microbiol 136, 481-487.

Archambault, D., Rondeau, P., Martin, D. \& Brodeur, B. R. (1991). Characterization and comparative bactericidal activity of mono. clonal antibodies to Bordetella pertussis lipooligosaccharide A. J Gen Microbin/ 137, 905-911.

Bauwens, J. E., Spach, D. H., Schacker, T. W., Mustafa, M. M. \& Bowden, R. A. (1992). Bordetella bronchiseptica pneumonia and bacteremia following bone marrow transplantation. J Clin Microbiol 30, $247+2475$.

Bradford, M. M. (1976). A rapid and sensitive method for the quantitation of microgram quantities of protein utilizing the principle of protein-dye binding. Anal Biocbem 72, 248-254.

Caroff, M. G. L. \& Karibian, D. (1990). Several uses for isobutyric acid-ammonium hydroxide solvent in endotoxin analysis. Appl Environ Microbiol 56, 1957-1959.

Caroff, M., Tacken, A. \& Szabo, L. (1988). Detergent accelerated hydrolysis of bacterial endotoxins and determination of the anomeric configuration of the glycosyl-phosphate present in the 'isolated Lipid A' fragment of the Bordetella pertussis endotoxin. Carbobydr Res 175, 273-282.

Caroff, M., Chaby, R., Karibian, D., Perry, J., Deprun, C. \& Szabo, L. (1990). Variations in the carbohydrate regions of Bordetella pertussi: lipopolysaccharides: electrophoretic, serological and structural features. J Bacteriol 172, 1121-1128.

Deprun, C., Karibian, D. \& Caroff, M. (1993). Analysis by ${ }^{252} \mathrm{Cf}$ plasma desorption mass spectrometry of Bordetella pertussis endotoxin after nitrous deamination. Int J Mass Spectrom Ion Proc 126, $187-190$.

Di Fabio, J. L., Caroff, M., Karibian, D., Richards, J. C. \& Perry, M. B. (1992). Characterization of the common antigenic lipopolysaccharide O-chains produced by Bordetella bronchiseptica and Bordetella parapertussis. FEMS Microbiol Lett 97, 275-282.

Girard, R. \& Chaby, R. (1985). Inhibition of lipopolysaccharide mitogenicity with characterized anti-lipid A monoclonal antibodies. Immunology 56, 481 487.
Goldstein, I. J., Hay, G. W., Lewis, B. A. \& Smith, F. (1970). Controlled degradation of polysaccharides by periodate oxidation, reduction and hydrolysis. Methods Carbohydr Chem 5, 361-370.

Goodnow, R. A. (1980). Biology of Bordetella bronchiseptica. Microbiol Rev 44, 722-738.

Johnson, K. G. \& Perry, M. B. (1976). Improved techniques for preparation of bacterial lipopolysaccharides. Can J Microbiol 22, 29-34.

Katzenstein, D. A., Ciofalo, L. \& Jordan, M. C. (1984). Bordetella bronchiseptica bacteremia. West J Med 140, 96-98.

Kersters, K., Hinz, K.-H., Hertle, A., Segers, P., Lievens, A., Siegmann, O. \& De Ley, J. (1984). Bordetella avium sp. nov., isolated from the respiratory tracts of turkeys and other birds. Int $J$ Syst Bacteriol 34, 56-70.

Le Dur, A., Chaby, R. \& Szabo, L. (1980). Isolation of two proteinfree and chemically different lipopolysaccharides from Bordetella pertussis endotoxin. $J$ Bacteriol 143, 78-88.

Lugtenberg, B., van Boxtel, R., van den Bosch, R., de Jong, M. \& Storm, P. (1984). Biochemical and immunological analyses of the cell surface of Bordetella bronchiseptica isolates with special reference to atrophic rhinitis in swine. Vaccine 2, 265-273.

Monack, D., Munoz, J. J. \& Peacock, M. G. (1989). Expression of pertussis toxin correlates with pathogenesis in Bordetella species. $J$ Infect Dis 159, 205-210.

Montaraz, J. A., Winter, A. J., Hunter, D. M., Sowa, B. A., Wu, A. M. \& Adams, L. G. (1986). Protection against Brucella abortus in mice with O-polysaccharide-specific monoclonal antibodies. Infect Immun 51, 961-963.

Musser, J. M., Hewlett, E. L., Peppler, M. S. \& Selander, R. K. (1986). Genetic diversity and relationships in populations of Bordetella species. J Bacteriol 166, 230-237.

Novotny, P. (1990). Pathogenesis in Bordetella species. J Infect Dis 161, 581-582.

Nowinsky, R. C., Lostrom, M. E., Tam, M. R., Stone, M. R. \& Burnette, W. N. (1979). The isolation of hybrid cell lines producing monoclonal antibodies against the $\mathrm{p} 15$ (E) protein of ecotropic murine leukemia viruses. Virologie 93, 111-126.

Pittman, M. (1984). The concept of pertussis as a toxin-mediated disease. Pediatr Infect Dis J 3, 467-486.

Rietschel, E. T., Schade, U., Jensen, M., Wollenweber, H. W., Lüderitz, O. \& Greisman, S. G. (1982). Bacterial endotoxins. Chemical structure, biological activity and role in septicemia. Scand $J$ Infect Dis 31, 8-21.

Simmons, D. G. \& Gray, J. G. (1979). Transmission of acute respiratory disease (rhinotracheitis) of turkeys. Avian Dis 23, 132-138.

Simmons, D. G., Gray, J. G., Rose, L. P., Dillman, R. C. \& Miller, S. E. (1979). Isolation of an etiologic agent of acute respiratory disease (rhinotracheitis) of turkey poults. Avian Dis 23, 194-203.

Terashima, M., Uezumi، I., Tomio, T., Kato, M., Irie, K., Okuda, T., Yokota, S. \& Noguchi, H. (1991). A protective human monoclonal antibody directed to the outer core region of Pseudomonas aeruginosa lipopolysaccharide. Infect Immun 59, 1-6.

Weiss, A. A. \& Hewlett, E. L. (1986). Virulence factors of Bordetella pertussis. Annu Rev Microbiol 40, 661-686.

Received 13 December 1993; revised 6 April 1994; accepted 13 April 1994. 